\title{
Adapting Participatory Design for Romanian Preschoolers Educational Software Development
}

\author{
Adriana-Mihaela Guran and Grigoreta-Sofia Cojocar \\ Babeş-Bolyai University, Cluj-Napoca, Romania \\ adriana@cs.ubbcluj.ro,grigo@cs.ubbcluj.ro
}

\begin{abstract}
The Participatory Design (PD) approach cannot be applied to developing software applications for small children (3-5/6 years) without considering the main constraint brought by their age: they cannot be involved in every step of the design. This paper presents our approach in adapting PD for preschoolers in the context of developing educational software that can be used as support for the teaching activities in Romanian kindergartens. We describe and discuss the results of evaluating the obtained software products with preschoolers and their teachers.
\end{abstract}

\section{Keywords}

Participatory Design · preschool children · educational software

\section{Context}

We live in a world that is more and more digital, and the children born nowadays are considered digital natives. This label can lead to the conclusion that the today generations possess the digital skills required by the European Union

How to cite this book chapter:

Guran, A-H. and Cojocar, G-S. 2020. Adapting Participatory Design for Romanian Preschoolers Educational Software Development. In: Loizides, F., Winckler, M., Chatterjee, U., Abdelnour-Nocera, J. and Parmaxi, A. (eds.) Human Computer Interaction and Emerging Technologies: Adjunct Proceedings from the INTERACT 2019 Workshops. Pp. 333-338. Cardiff: Cardiff University Press. DOI: https://doi. org/10.18573/book3.aq. License: CC-BY 4.0. 
labor market of the future, when $90 \%$ of jobs will require digital skills. The European Union statistics on digital skills rank Romania on the last position, from 28 countries, in the last 2 years [5]. An effective intervention would necessarily target education. In Romania, ICT is studied from the primary school until the end of the mandatory education. We propose a shift in the classical approach of introducing computer skills, by using technology to learn, rather than teaching children how to use technology. Our approach tries to go even further, by providing support in developing the digital skills of preschoolers (3 to $5 / 6$ years) in the form of tools (interactive applications) that can be used in the public formal preschool education system during the teaching activities. Our initiative needs to achieve two goals: being educative (such that kindergarten teachers want to use it) and being entertaining (such that the children want to interact with). Such goals cannot be achieved without focusing our design on children and kindergarten teachers. Thus, we have considered that we need to involve both categories in the design process. Although we have experience in PD with adults, the challenge is to keep the focus on the final users (the children) while respecting the constraints imposed by the client (the kindergarten teachers).

\section{Procedure \& Results}

Although a large number of design guidelines for children have been proposed $[2,6-7]$, little attention is given to designing for preschoolers. Recent studies $[3,8]$ show that most of the applications consider the children aged 0 to 8 being a homogeneous group. They also suggest that the interaction techniques and content are not adapted to children development. The main differences between preschool and school children are that preschool children cannot read or write, they cannot complete adults stated tasks without being rewarded, and their main activity is playing. All these differences add new constraints on the design of interactive applications for preschool children: the applications should be conceived as games or at least they should expose games-related characteristics in order to be suitable, they should not use written output and they should not require written input. The interaction of the children with the applications should require basic (fundamental) computer skills: pressing a key on the keyboard (space, enter, arrows), moving the cursor on the screen or clicking. The content provided by the applications should also align with the Curriculum for preschool children from Romania. In order to keep our focus on the final users, we have decided to apply PD and to involve the final users during the design and development as much as possible. We knew from the beginning that children can not be involved in every stage of the design process because of the cognitive constraints imposed by their age. Still, we have tried to keep them present in our approach by means of the kindergarten teacher which played the role of a surrogate (proxy) of our real users. We 
considered it a good strategy as the kindergarten teacher has in-depth knowledge about children development, their cognitive and physical capabilities and limitations, and the appropriate learning goals for their age. In order to design and develop the intended educational applications, we have benefited the participation of Computer Science students from our faculty attending the HCI elective course. One of the main goals of this class is to make the students understand the importance and benefits of PD. They worked in teams of 3 to 5 members. The teams have started the PD process with an initial meeting with the client (the kindergarten teacher) who briefly described her need for interactive applications as support in her classic teaching activities, the environment where she works and how the teaching activities take place at that moment. She also specified initial requirements for each team containing the age range of the users, the general theme, the integrated curricula domains and some task examples (e.g. theme: The fall (children aged 3-4); integrated domains: Language and Communication and Physical Education; tasks: Poems, rhymes, a story about leaves; Verbal tasks: such as We turn around, we kneel at once, a yellow leaf we hope to find). She specified that the applications should consider all the stages in the teaching process: focus capturing, content presentation, and fixation game. Afterwards, students have participated to observation sessions in the kindergarten with the goals of meeting the final users and finding information about children knowledge of the domain and their digital skills. This way, students realized that most of their initial ideas about preschoolers were wrong. They thought children are capable of reading, they supposed children can perform click, double-click, or drag and drop operations. But the reality was, that, in some cases, children could not even hold a mouse on their hands (the mouses were physically too big for their small hands). As such, during the requirements, we have involved the kindergarten teacher as client and the preschoolers as informants. In the second step, students have created design alternatives and the kindergarten teacher has provided feedback regarding the presented content, interaction, and proposed tasks. It was the only step where children haven't been directly involved, but they have been represented by their surrogate. She played the role of the children in terms of answering to the proposed tasks and commented on the presented content simulating the children reaction to them. In the third step the high fidelity executable prototypes have been developed taking into consideration the feedback from the kindergarten teacher. The teams have visited again the children in the kindergarten to gather feedback on the prototypes. Children have participated as users and testers of the applications. Comments of the children regarding the characters and objects on the application have been then transposed into design decisions. The evaluation sessions were organized as play-testing sessions with individual users. The kindergarten teacher and the students observed the children freely exploring the application. After that, short post-interviews to reveal the subjective opinion of children have been organized. They consisted in simple questions, such as: Would you like to play/show this game with/to your friends? Some teams have decided to use peer tutoring to simulate 
think aloud protocols, and others have used smileyometers [10] to help children express their attitude toward the applications. This step has brought more unexpected information regarding the way preschool children understand the interaction. The most frequent problem was related to how the tasks were stated. For example, if the children were required to choose the objects on the screen having a certain property, they were always using their finger to indicate them. Afterwards, the indications were explicitly reformulated by select with a click statements. Also, the children had frequent questions about the tasks they should perform, how do they go back to a previous screen or how they can exit. The solution for this type of problems was to introduce characters that greet the children in the beginning, and guide them through the interaction (providing interaction support). The results of the usability testing sessions were encouraging, as the children were willing to use again and again the assessed application. We have validated the results of the usability testing by applying a preschooler adapted HECE (Heuristic Evaluation of Child E-Learning) [1] with 12 expert users (other kindergarten teachers) for ten applications. Each application was assessed by two evaluators. Seven out of ten applications were considered successful related to the HECE heuristics. The other three applications presented problems on the NUH component from HECE (navigation related heuristics). Two applications had problems on the CUH component (children skills) that were related to the use of abstract concepts, that are too difficult to understand by the children. The learning component (LUH) has been evaluated with the highest scores, due tot the active participation of an expert in the design process.

\section{Discussion}

In this paper we have presented an approach of using participatory design with Romanian preschool children. We have involved the preschoolers in almost all design phases: during requirements as informants, during prototyping as users and informants, and during evaluation as users and testers. The only step where they haven't been involved was the initial design, where the design sketches were too abstract to be evaluated by children, but their surrogate has successfully replaced them. The results of usability testing and validation testing show us that preschoolers can be used as informants, users and testers during the design process. Potentially, they could play a more significant role as design partners, but only if they are accompanied by an expert in the educational field.

\section{References}

1. Asmaa A. and Asma Al-O. Usability heuristics evaluation for child e-learning applications. In Proceedings of the 11th International Conference on Information Integration and Web-based Applications \& Services (iiWAS '09). ACM, New York, NY, USA, 425-430. 
2. Bekker, T., and Markopoulos P. Interaction design and children. In:Interacting with Computers 15 (2003)

3. Crescenzi, L., and Gran, M. An Analysis of the Interaction Design of the Best Educational Apps for Children Aged Zero to Eight. Comunicar, 46, 77-85. doi: https://doi.org/10.3916/C46-2016-08

4. European Commission, Digital Single Market,2017, retrieved from https://ec .europa.eu/digital-single-market/

5. Europe's Digital Progress Report (EDPR) 2018, How digital is your country? Europe needs Digital Single Market to boost its digital performance, retrieved from https://ec.europa.eu/digital-single-market/en/news/how -digital-yourcountry-europe-needs-digital-single-market-boost-its-digital -performance

6. Fails, A., Guha, M. L., and Druin, A. Methods and Techniques for Involving Children in the Design of New Technology for Children. Hanover, MA, USA: Now Publishers Inc., 2013.

7. Hourcade, J. P. Interaction Design and Children Found. Trends Hum.Comput. Interact. 1, 4 (April 2008), 277-392.

8. Kazakoff, E. Technology-based literacies for young children: Digital literacy through learning to code. In K.L. Heider \& M.R. Jalongo (Eds), Children and Families in the Information Age: Applications of Technology in Early Childhood (pp. 43-60). New York: Springer, (2015)

9. Prensky, M. Digital Natives, Digital Immigrants, On the Horizon 9(5): 1-6, doi: https://doi.org/10.1108/10748120110424816 2001.

10. Read, J. C., and MacFarlane, S. Using the fun toolkit and other survey methods to gather opinions in child computer interaction. In Proceedings of the 2006 conference on Interaction design and children (IDC '06). ACM, New York, NY, USA, 81-88.(2006)

11. Sefton-Green, J., et al. Establishing a Research Agenda for the Digital Literacy Practices of Young Children: a White Paper for COST Action IS1410. 2016. 